\title{
Cloud history changes water-ice-surface interactions of oxide mineral aerosols (e.g. Silica)
}

Ahmed Abdelmonem ${ }^{1 *}$, Sanduni Ratnayake ${ }^{2}$, Jonathan D. Toner ${ }^{3}$ and Johannes Lützenkirchen ${ }^{2}$

${ }^{1}$ Institute of Meteorology and Climate Research - Atmospheric Aerosol Research (IMKAAF), Karlsruhe Institute of Technology (KIT), 76344 Eggenstein-Leopoldshafen, Germany

${ }^{2}$ Institute of Nuclear Waste Disposal (INE), Karlsruhe Institute of Technology (KIT), 76344 Eggenstein-Leopoldshafen, Germany

$10{ }^{3}$ Department of Earth \& Space Sciences, University of Washington, Seattle, WA 98195, USA

* Correspondence to: A. Abdelmonem (ahmed.abdelmonem@kit.edu) 


\section{S1 Thermodynamic considerations}

Calculations have been done using and data pertaining to silica (Marion et al., 2009)combined with the the frezchem database included in PHREEQC Interactive, version 3.4.0.12927 (released November 9, 2017). The calculations were done for the experimental conditions at room temperature in terms of solution composition. Two extreme cases were considered:

5 Ice formation and no ice formation. When including the formation of ice, super-cooling cannot be simulated. In this case the $\mathrm{pH}$ drops in the $\mathrm{H}_{2} \mathrm{O}$ fraction that is not freezing because the solutes are concentrated in remaining liquid that remains due to the freezing point depression. Since in the experiments in no case all of the water freezes, the calculations overestimate the effect. The final calculated volume of liquid water at $-40^{\circ} \mathrm{C}$ is about $300 \mu \mathrm{L}$, which contains all the solutes. In this case the $\mathrm{pH}$ drops to about -1.4. In our experiments, the freezing and melting is occurring at the surface. So extreme conditions could occur during the melting only, when remaining solution connects to the interfacial water film, but as pointed out above in all cycles much larger volumes of liquid water are expected compared to the $300 \mu \mathrm{L}$ noted above. The amount of dissolved silica as a function of the temperature at equilibrium is shown in Figure S1 as a dotted line. The equilibrium concentration of silica at $-40^{\circ} \mathrm{C}$ is about $100 \mu \mathrm{M}$ based on the available thermodynamic data.

When excluding the formation of ice in the calculation as the other extreme, the water remains liquid and slightly changes its volume, which affects molar concentrations accordingly. The $\mathrm{pH}$ remains more or less constant in this case. The concentration of silica at $-40^{\circ} \mathrm{C}$ is about $410 \mu \mathrm{M}$, i.e. a factor 4 higher than in the previous case. This is due to the higher salt concentration in the previous example, where the activity coefficients of the solutes cause a decrease in the solubility.

The measured concentration of silica in the experiments was about $41 \mu \mathrm{M}$.

The measurements and observations can be related to the above calculations as follows:

- The measured silica concentration (at the end of the experiment at TP cycle 25) is below any of the estimates for equilibrium solubility for the conditions of our experiments. This means that there is a driving force for further dissolution. This corroborates that the number of cycles is the major factor and not the time of exposure, since the prolonged time of exposure at room temperature (i.e. TP > 25) should have caused more dissolution and thus more SHG enhancement. Instead the freeze/melt cycles cause the changes in SHG since with lowering temperature.

- The lower silica solubility, with decreasing temperature, should favor the interaction of dissolved silica with the fused silica surface. Based on the work by (Schaefer et al., 2018), this corroborates the idea that there is a trend to silica adsorption with the decreased temperature. There is ample information for example that dissolved $\mathrm{Al}$ adsorbs to aluminium oxide and our experiments with dissolved silica in the presence of fused silica also shows such interaction, so that the temperature dependence in terms of silica solubility would corroborate our interpretation in the main text. 


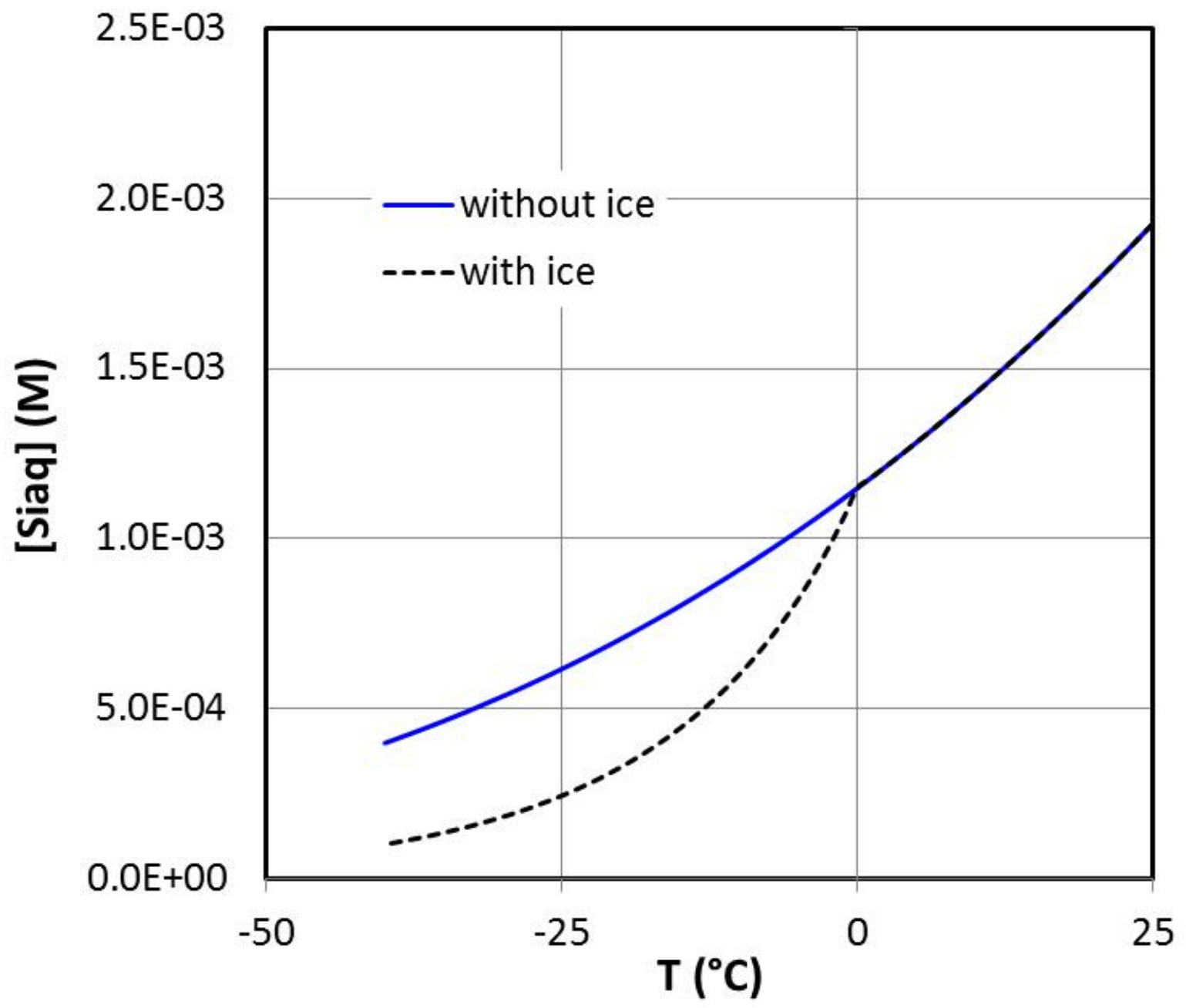

Figure S1: Evolution of dissolved silica in a solution of $1 \mathrm{mM} \mathrm{HCl} \mathrm{(pH} 3$ at room temperature) with decreasing temperature in the presence of ice formation (dotted line) and without ice forming (full line). Calculations were done as specified in the text. 
S2 Comparison between PM and SM polarization combinations under the geometrical conditions of the used SHG setup

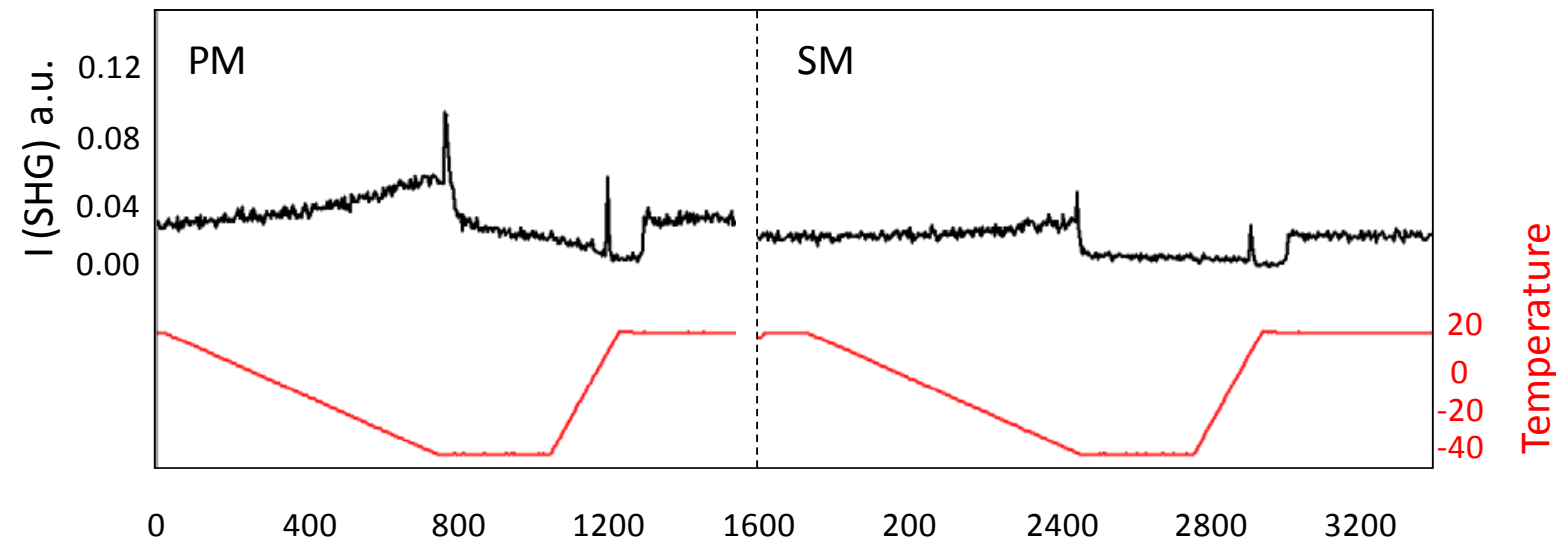

5 Figure S2: SHG signal (rescaled raw data) at the aged pH3 solution-silica interface as a function of time/temperature for two successive TP cycles with different beam polarization combinations (PM: P-polarized SHG / 45-polarized incident, and SM: S-polarized SHG / 45polarized incident) incident with an angle close to the critical angle of total internal reflection. An aged sample was used to minimize the sample surface change during the two runs. The data show that the signal versus temperature for both polarization combinations behaves identically. 

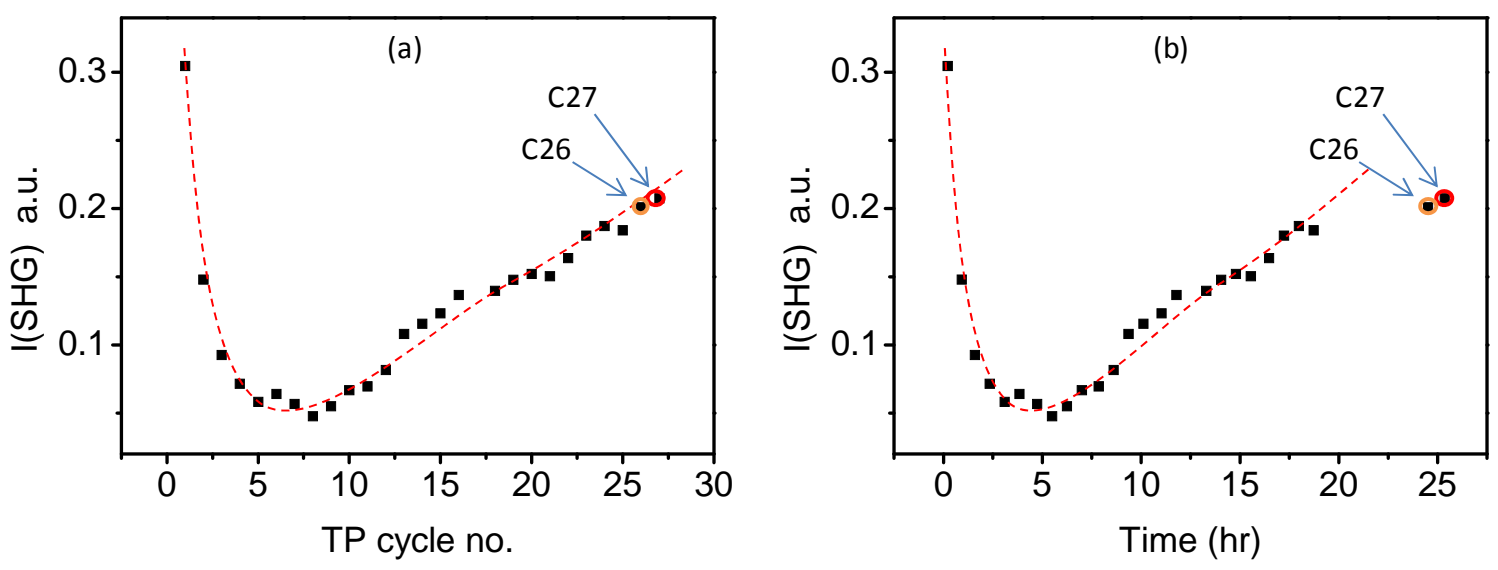

Figure S3: SHG signal at pH3 solution-silica interface as a function of TP cycle number (a) and time (b) of liquid signal at $20{ }^{\circ} \mathrm{C}$ during 5 repeating the freezing-melting TP. The dashed red lines are trend lines. CS26 and CS27 denote data points that lie on the trend line in (a) but not in (b). This shows that the significant aging we observe in this work arises from the freezing-melting process and not from the time the sample being in contact with solution. Furthermore, as discussed in section S1, the measured silica concentration should favor further dissolution at room temperature, but this does not explain the SHG data. 


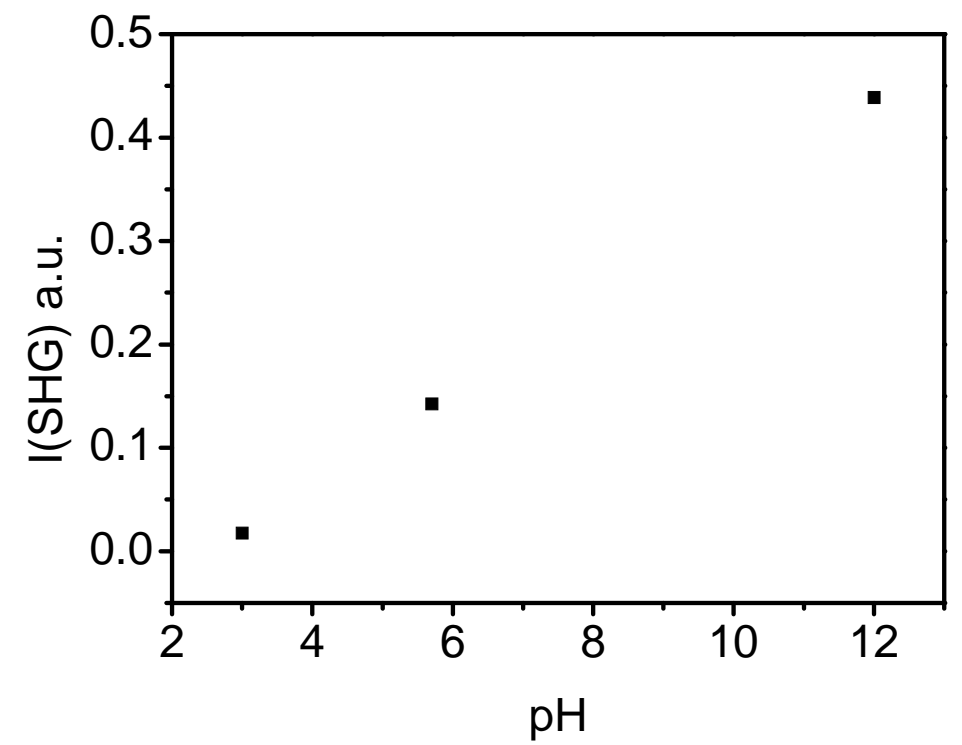

Figure S4: SHG signal as a function of $\mathrm{pH}$ for our fresh sample (before aging) at $20^{\circ} \mathrm{C}$. 


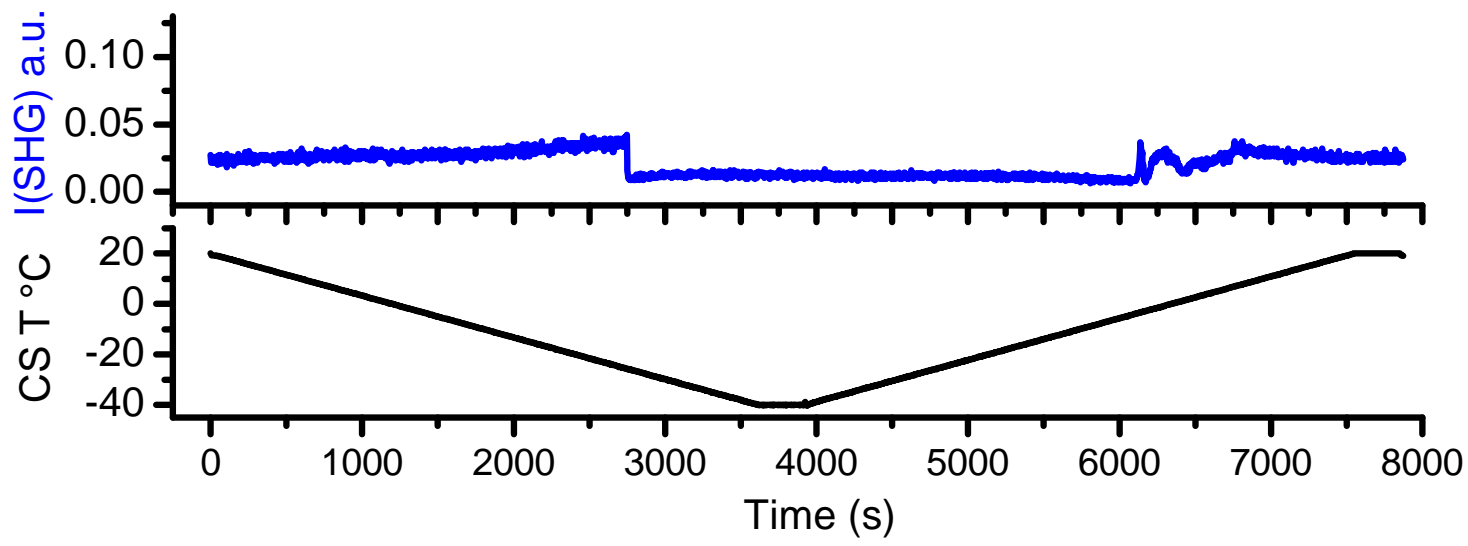

Figure S5: SHG signal at pH3 solution-silica interface as a function of time for the C27 run which was carried out at cooling and heating 5 rates $\left(=1^{\circ} \mathrm{C} / \mathrm{min}\right)$ slower than the standard TP. 


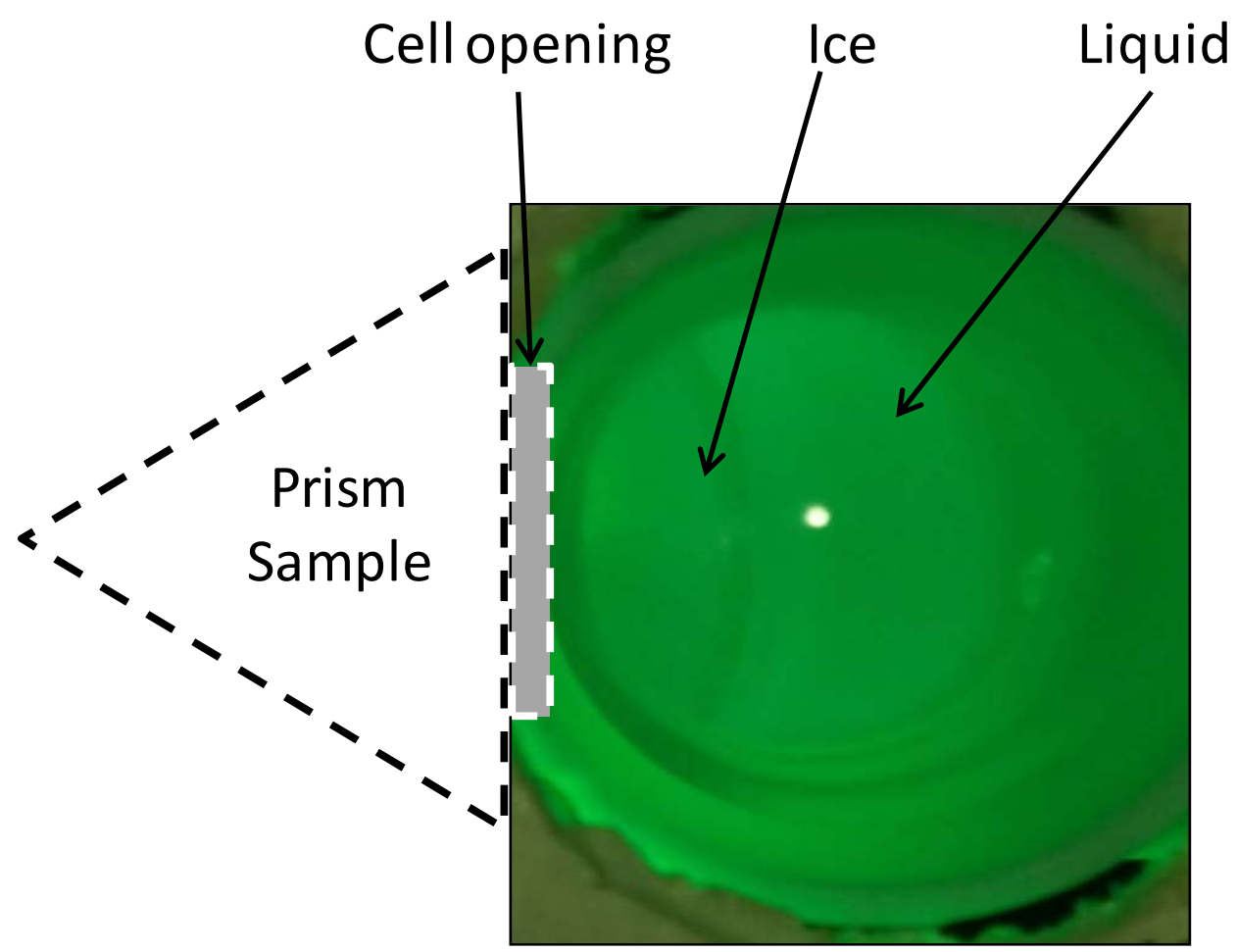

Figure S6: Photo (top view) of the bulk ice formed after freezing and waiting for $5 \mathrm{~min}$ at $-40{ }^{\circ} \mathrm{C}$. The freezing starts at the surface of the sample and grows at the expense of water on the other side. The size of the bulk ice is proportional to the time of keeping the system 5 supercooled after the freezing event. The ice piece is stuck in the cell opening and the process of melting and departing from the sample neighborhood depends on the cell geometry and bulk ice size as well as the thermal conditions. All these parameters affect the appearance and disappearance of the confined liquid signal.

\section{References}

10 Marion, G. M., Crowley, J. K., Thomson, B. J., Kargel, J. S., Bridges, N. T., Hook, S. J., Baldridge, A., Brown, A. J., Ribeiro da Luz, B., and de Souza Filho, C. R.: Modeling aluminum-silicon chemistries and application to Australian acidic playa lakes as analogues for Mars, Geochimica et Cosmochimica Acta, 73, 3493-3511, doi: https://doi.org/10.1016/j.gca.2009.03.013, 2009.

Schaefer, J., Backus, E. H. G., and Bonn, M.: Evidence for auto-catalytic mineral dissolution from surface-specific vibrational spectroscopy, Nat. Commun., 9, 3316, doi: 10.1038/s41467-018-05762-9, 2018. 